\title{
Design and test of soft-pot-tray automatic embedding system for light-economical pot seedling nursery machine
}

\author{
Lintao Chen ${ }^{1}, \mathrm{Xu} \mathrm{Ma}^{1 *}$, Cong Wang ${ }^{1}$, Hongwei $\mathrm{Li}^{1}$, Zehua $\mathrm{Li}^{2}$, Xueshen Chen ${ }^{1}$, Tao Chen ${ }^{1}$ \\ (1. College of Engineering, South China Agricultural University, Guangzhou 510642, China; \\ 2. College of Mathematics and Informatics, South China Agricultural University, Guangzhou 510642, China)
}

\begin{abstract}
Most of the commercially-available pot seedling nursery machines are incompatible with soft-pot-trays and are labor-intensive and low in productivity. A soft-pot-tray automatic embedding system was developed in this study to achieve automatic embedding of the soft pot tray into the hard tray following sowing and covering with soil. A control system was constructed using the Arduino program development environment. An embedded-hard-tray automatic lowering mechanism and conveyor-belt-based pot-tray embedding system were designed. Dynamics analysis was conducted to derive an equation to describe the embedding process of the soft pot tray into the embedded hard tray. A prototype of the soft-pot-tray automatic embedding system was manufactured and tested. The analytical equation suggested that a minimum linear velocity of $0.86 \mathrm{~m} / \mathrm{s}$ was required for a complete embedding process. The experimental results showed that the embedded-hard-tray automatic lowering mechanism was reliable and stable as the tray placement success rate was greater than $99 \%$. The successful tray embedding rate was $100 \%$ and the seed exposure rate was less than $1 \%$ with a linear velocity of the conveyor belt of $0.92 \mathrm{~m} / \mathrm{s}$. The experiment findings agreed well with the analytical results. The proposed soft-pot-tray automatic embedding system satisfied the technical specifications for a light-economical pot seedling nursery machine.
\end{abstract}

Keywords: automatic embedding, pot seedling nursery machine, embedded hard tray, soft pot tray, conveyor-belt embedding system

DOI: $10.25165 /$ j.ijabe.20201301.4726

Citation: Chen L T, Ma X, Wang C, Li H W, Li Z H, Chen X S, et al. Design and test of soft-pot-tray automatic embedding system for light-economical pot seedling nursery machine. Int J Agric \& Biol Eng, 2020; 13(1): 91-100.

\section{Introduction}

Rice is one of the principal grain crops in China. However, rice planting mechanization remains underdeveloped in the rice paddy mechanization system ${ }^{[1-3]}$. Currently, rice paddy planting is mainly performed by raising seedling and transplantation. Mechanized rice paddy seedling transplantation can be either the blanket seedling or the pot seedling transplantation. The pot seedling transplantation has many advantages, such as minimum root injury, quick regreening, early tillering, and suitability for a wide range of seedling age $\mathrm{e}^{[4,5]}$. It also avoids some of the problems of traditional machine transplanting and therefore increases the yield potential. Recent studies on mechanized rice paddy planting were focused on the pot seedling transplantation ${ }^{[6]}$. The development of pot seedling nursery machines is necessary to achieve automated seedling transplantation ${ }^{[7-11]}$. However, commercially available seedling nursery machines are only applicable to hard pot trays, which are cost prohibited. Therefore,

\section{Received date: 2018-10-19 Accepted date: 2019-12-24}

Biographies: Lintao Chen, $\mathrm{PhD}$ Candidate, research interests: agricultural mechanization and automation, Email: 1574287180@qq.com; Cong Wang, Graduate student, research interests: agricultural mechanization and automation, Email: 865138312@qq.com; Hongwei Li, PhD Candidate, research interests: agricultural mechanization and automation, Email: 1592145856@qq.com; Zehua Li, PhD, Associate Professor, research interests: agricultural mechanization and automation, Email:499927441@qq.com; Xueshen Chen, $\mathrm{PhD}$, Associate Professor, research interests: agricultural mechanization and automation, Email: 26565410@qq.com; Tao Chen, Graduate student, research interests: agricultural mechanization and automation, Email: 1543287208@qq.com.

*Corresponding author: Xu Ma, PhD, Professor, research interests: agricultural mechanization and automation College of Engineering, South China Agricultural University, Guangzhou 510642, China. Tel: +8613560008927, Email: maxu1959@scau.edu.cn. it is desired to develop a pot seedling nursery machine for soft pot trays. A pot seedling nursery machine usually performs multiple tasks from soil-bed setting to soil-bed compaction to precise sowing to soil coverage and finally clean-up ${ }^{[12]}$.

To achieve soft-pot-tray operations such as soil-bed compaction and precise sowing, pot seedling nursery machines utilize synchronous drive of chains and steel wires for precision position control. The position-controlling method matches the chain pitch with the center distance of the holes for the soft pot tray ${ }^{[13,14]}$. The advantages of this method are that the structure is simple and its operation is reliable. The disadvantage is that workers have to carry the hard trays, waiting for soft-pot-tray embedding, and then perform setting operations after completing seed sowing. As a result, it is very labor-intensive and low productivity. Therefore, a soft-pot-tray automatic embedding system for a pot seedling nursery machine is required.

To embed soft pot trays into hard trays automatically, it is necessary to design an automatic hard-tray-providing system as well as a soft-pot-tray conveyor and embedding system. No studies have been found on the embedding of soft pot trays into hard trays. However, similar studies have reported automatic tray-providing mechanisms and apparatuses for hard trays. The US and some European countries have developed good quality, highly automatic seed nursery machines such as Blackmore System, Speedling System, Marksman, and Hamilton for economic crops such as vegetables and flowers ${ }^{[15-19]}$. They also designed automatic nursery-tray-providing devices of hard trays that supply trays by a combination of pneumatic and mechanical principles. Iseki Co., Ltd. and Kubota Agriculture Machinery Co., Ltd. have developed various automatic tray-providing devices for hard trays with different structures such as spiral, guide rods, and swinging 
$\operatorname{arms}^{[20,21]}$. Yixiang Co. Ltd. developed a pneumatically driven automatic tray-providing device ${ }^{[22]}$. These devices have highly sophisticated structures and complex control systems and are mainly used for crops with high economic value. Moreover, they can only be used with special trays. These automatic tray-providing devices provide an important reference for our research. However, a pneumatically driven automatic tray-providing device is costly and it generates vibration during operation, which is undesired for seedling transplantation. To solve these problems, Ma Xu et al. ${ }^{[23]}$ developed an electronically controlled hard and soft trays automatic providing system which used a STM 32 micro-controller as the control core to overcome the problems. They also developed an embedded hard tray, which solved the problems of poor stacking stability of the hard trays and easy separation of the soft trays from the hard trays during automatic tray-providing operation. The embedded hard trays and electronically controlled tray-providing system play a prominent role in soft-pot-tray automatic embedding system.

In order to achieve automatic embedding of the soft pot tray into the hard tray after sowing and covering the soil, we designed a soft-pot-tray automatic embedding system to facilitate the subsequent setting operations and automatic stacking of the soft pot trays. In this study, dynamics analysis was used to obtain the minimum linear velocity needed of the conveyor belt for the complete embedding of the soft pot tray into the hard tray. A stable and reliable control system was constructed in Arduino program development environment. An embedded-hard-tray automatic lowering mechanism and a conveyor-belt soft-pot-tray embedding system were developed. Finally, a principle prototype was manufactured and experiments were conducted to validate the theoretical analysis and evaluate the structure, working conditions, and main parameters of the soft-pot-tray automatic embedding system.

\section{Materials and methods}

\subsection{Structure of soft-pot-tray automatic embedding system}

As shown in Figure 1, the length, width, and height of soft-pot-tray automatic embedding system for a light-economical pot seedling nursery machine are $3000 \mathrm{~mm}, 500 \mathrm{~mm}$, and $700 \mathrm{~mm}$ respectively. During operation, the soft-pot-tray automatic embedding system is placed at the back of the seedling nursery machine. The embedding system comprises a frame, a conveyor-belt electric motor $M_{1}$, a transport mechanism driven by electric motor $M_{2}$, a position-limiting mechanism, a conveyor-belt embedding mechanism, detection switches $A$ and $B$, an embedded-hard-tray automatic lowering mechanism (including an electrical steering gear, a lifting shaft, and a lifting head), a detection protection switch $C$, a transport mechanism, and an electrical control box.

The operating process is as follows. A stack of embedded hard trays (normally comprising 10 trays) is placed in the embedded-hard-tray automatic lowering mechanism. The operation switch in the electrical control box is activated to restore the steering gear to its original position, with the lifting-head starting point at zero. The motor $M_{1}$ controls the movement of the conveyor-belt embedding mechanism and prevents the motor $M_{2}$ from operating; in other words, the transport mechanism does not operate. When the soft pot tray with sown seeds touches the detection switch $A$, the electrical steering gear is activated. This turns the lifting shaft and drives the lifting head to raise all trays from the lowest-level embedded hard tray upward. Therefore, the control on both sides of the position-limiting mechanism and the conveyor-belt embedding mechanism functions are integrated. Furthermore, the soft pot tray is completely embedded in the lowest-level embedded hard tray. At this stage, detection switch $B$ detects the end of the soft pot tray and drives $M_{2}$ to start the transport mechanism, and it continues to transport the embedded hard tray with the embedded soft pot tray. Follow-up workers take the tray and place it into the automatic stacked-tray device for stacking. At the same time, detection protection switch $C$ detects embedded hard trays as well as soft pot trays. This guarantees that the seedling trays fully exit the embedded-hard-tray automatic lowering mechanism. Then, the electrical steering gear operates again to drive the lifting head to lower the additional accumulated embedded hard trays. This completes one soft-pot-tray automatic embedding operation. Once the next soft pot tray arrives, the above process is repeated to perform continuous operation. It should be noted that to prevent the late arrival of follow-up embedded hard trays, workers must load the embedded hard trays in a timely manner.
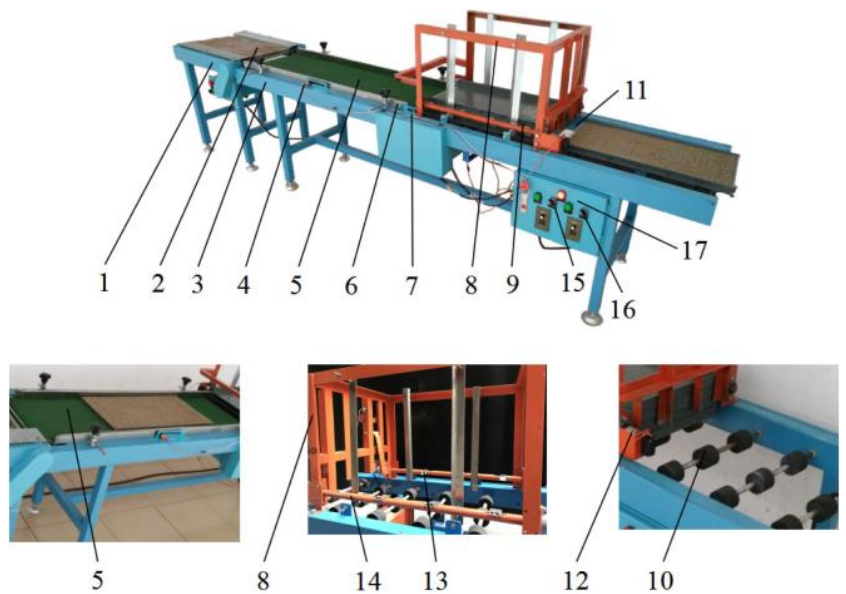

1. Back end of pot seedling nursery machine 2. Soft pot tray with sown seeds 3. Frame 4. Detection switch $A$ 5. Conveyor-belt embedding mechanism 6. Position-limiting mechanism 7. Detection switch $B$ 8. Embedded-hard-tray automatic lowering device 9. Stack of embedded hard trays 10. Transport mechanism 11. Detection protection switch $C$ 12. Electrical steering gear 13. Lifting head 14. Lifting shaft 15. Transport mechanism driven by electric motor $M_{2}$ 16. Electrical control box 17 . Transportation electric motor $M_{1}$

Figure 1 Structural schematic of soft-pot-tray automatic embedding system

\subsection{Soft pot trays and embedded hard trays}

The internal length, width, and height of soft pot trays are 580, 280 , and $25 \mathrm{~mm}$ respectively and a tray has 406 holes (14 rows by 29 columns, Figure $2 \mathrm{a})$. The embedded hard trays have internal dimensions of $600 \mathrm{~mm}(l) \times 300 \mathrm{~mm}(w) \times 30 \mathrm{~mm}(h)$. They are made of polyvinyl chloride (PVC) that is pressed into shape to form the tray bottom and three sides (Figure 2b). Figure 2c shows photographs of the soft pot tray with sown soil embedded into the embedded hard tray.

\subsection{Design and dynamics analysis of conveyor-belt embedding mechanism}

A preliminary test of soft-pot-tray automatic embedding system showed that the steel-wire-driven transport effectively transported the soft pot trays (Figure 3a), with the chain position axis controlling the soft-pot-tray position and the chain driving the soft pot trays to move synchronously ${ }^{[13]}$. However, as the soft-pot-tray bottom is always in contact with the steel wire, the tray may have insufficient follow-through force. In this case, the soft pot tray would not be fully embedded into the embedded hard 
tray, and the end section of the former would jut out of the embedded-hard-tray end by a distance of $L_{0}(\mathrm{~m})$, see Figure $3 \mathrm{~b}$.

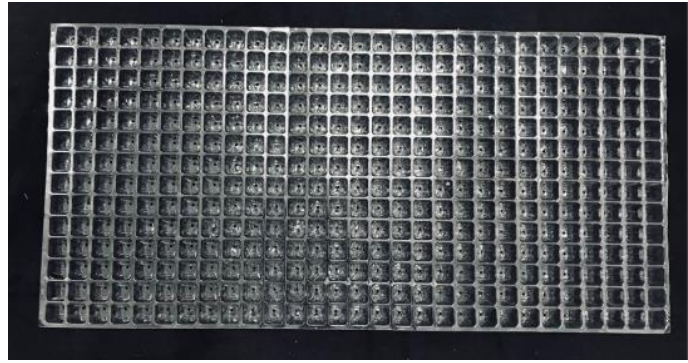

$$
\text { a. Soft pot tray }
$$

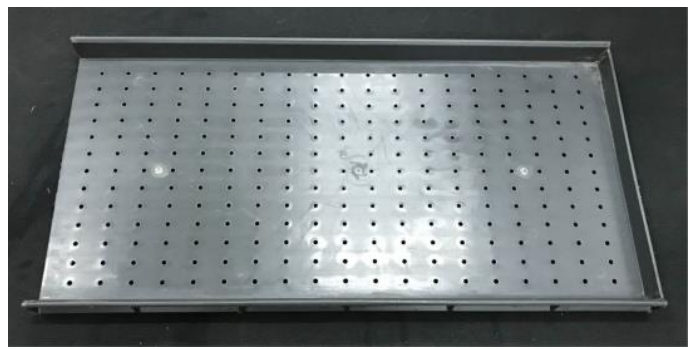

b. Embedded hard tray

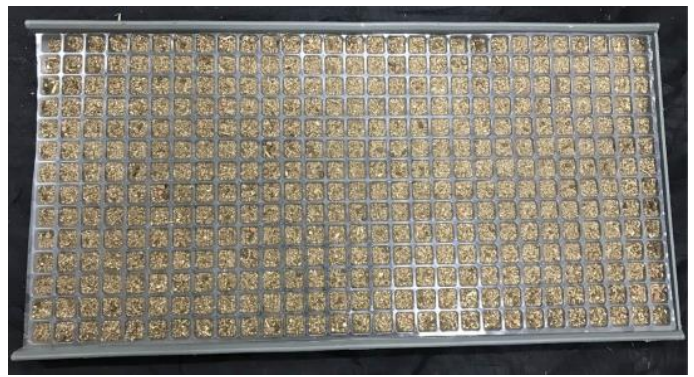

c. Soft pot tray with sown soil embedded into an embedded hard tray

Figure 2 Photographs of soft pot trays and embedded hard trays

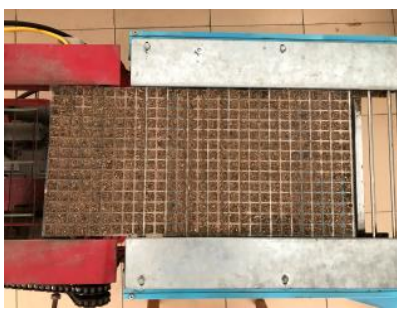

a. Steel wire chain used for transporting soft pot tray

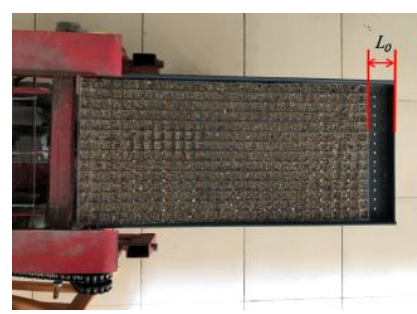

b. Soft pot tray with sown soil embedded into an embedded hard tray
Figure 3 A preliminary test of soft-pot-tray automatic embedding

Therefore, to ensure that soft pot trays with sown seeds are completely embedded into the embedded hard trays, the end of the steel-wire-driven transport component must have a conveyor-belt embedding mechanism with a certain length and smooth acceleration. The total soft-pot-tray embedding speed is influenced by the linear speed $V_{0}(\mathrm{~m} / \mathrm{s})$ of the conveyor belt. Therefore, dynamics analysis was conducted in this study to determine the appropriate $V_{0}$. Figure 4 shows schematics of soft pot trays being embedded into the embedded hard trays.

Initially, no sliding occurs between the soft pot tray and conveyor belt as the soft pot tray moves with the conveyor belt (Figure 4a). Thus, the conveyor belt $V_{0}$ is equal to the speed of the soft pot tray (also $V_{0}$ ). As the soft pot tray is gradually embedded into the embedded hard tray under the effect of the static friction of the conveyor belt, $V_{0}$ is initially maintained by the tray. However, when $F_{2}(N)$, the fiction resistance received by the soft pot tray from the embedded hard tray, exceeds the maximum static friction received by the soft pot tray from the conveyor belt, $F_{1}(N)$, sliding occurs between the soft pot tray and conveyor belt, causing a speed reduction. Figure $4 \mathrm{~b}$ shows a force analysis of the soft pot tray at this stage.

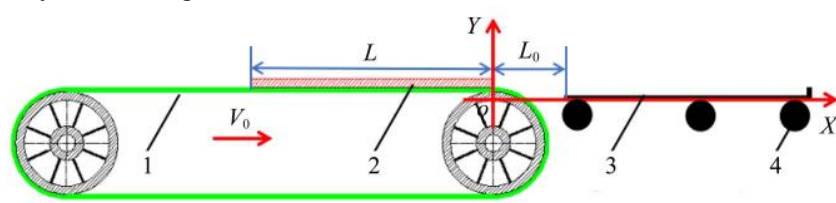

a.

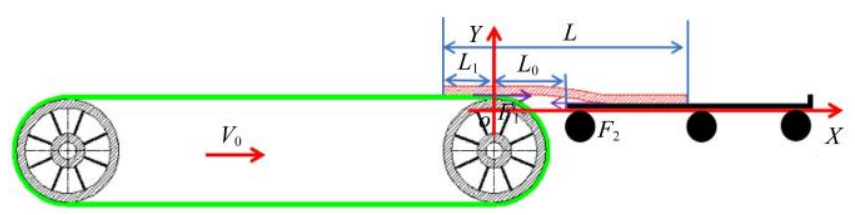

b.
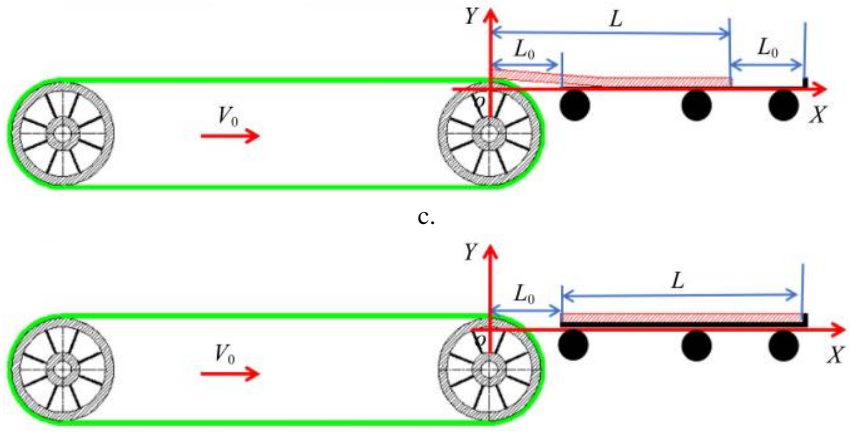

(a)-(d) Various process stages. 1. Conveyor belt 2. Soft pot tray 3. Embedded hard tray 4. Rubber roller

Figure 4 Schematics of soft pot tray being embedded into embedded hard tray

For simplifying the analysis, the soft-pot-tray body was assumed to be an evenly distributed continuum with equal properties at all points. The distance between the end point of the conveyor-belt embedding machine and the embedded-hard-tray start point was labeled $L_{0}(\mathrm{~m})$. For analysis with the $X$ direction being vertical to the soft pot tray, the mass and total length were labeled as $M(\mathrm{~kg})$ and $L(\mathrm{~m})$, respectively. Therefore, $F_{1}$ can be expressed as follows:

$$
F_{1}=f_{1} L_{1}=\mu_{1}\left(\frac{M}{L}\right) g L_{1}
$$

where, $f_{1}$ is friction on length of the conveyor-belt unit received by soft pot tray, $\mathrm{N} / \mathrm{m} ; L_{1}$ is the length of belt occupied by soft pot tray, $\mathrm{m} ; \mu_{1}$ is the maximum coefficient of static friction between the conveyor belt and soft pot tray.

Therefore, $F_{2}$ is expressed as

$$
F_{2}=f_{2}\left(L-L_{0}-L_{1}\right)=\mu_{2}\left(\frac{M}{L}\right) g\left(L-L_{0}-L_{1}\right)
$$

where, $f_{2}$ is friction received from embedded hard tray on unit length of soft pot tray, N/m; $L-L_{0}-L_{1}$ is lengths of soft pot tray in embedded hard tray and on conveyor belt (m) (see Figure $4 \mathrm{~b}$ ); $\mu_{2}$ is the sliding friction coefficient between embedded hard tray and soft pot tray.

To simplify the calculations, $\mu_{1}$ and $\mu_{2}$ were assumed to be equal. At this stage, $F_{1}$ and $F_{2}$ are identical; thus, we obtain the following from Equations (1) and (2):

$$
L_{1}=\frac{\mu_{2}}{\mu_{1}+\mu_{2}}\left(L-L_{0}\right)
$$

From the point where speed reduction begins until the position shown in Figure $4 c$, if the soft pot tray leaves the conveyor belt 
with speed $V_{1}(\mathrm{~m} / \mathrm{s})$, we obtain the following from the kinetic energy theorem:

$$
\frac{1}{2} M V_{0}^{2}-\frac{1}{2} M V_{1}^{2}=-A_{1}+A_{2}
$$

where, $A_{1}$ is positive work from soft pot tray and conveyor-belt friction at distance $L_{1}, \mathrm{~J} ; A_{2}$ is negative work from soft pot tray and conveyor-belt friction at distance $L_{1}, \mathrm{~J}$.

From Equation (5), the following relations can be derived:

$$
\begin{gathered}
A_{1}=\int_{0}^{L_{1}} f_{1} x d x=\frac{1}{2} \frac{M}{L} \mu_{1} g L_{1}^{2} \\
A_{2}=\int_{L-L_{0}-L_{1}}^{L-L_{0}} f_{2} x d x=\frac{1}{2} \frac{M}{L} \mu_{2} g\left(2 L-2 L_{0}-L_{1}\right) L_{1}
\end{gathered}
$$

As shown in Figure $4 \mathrm{~d}$, if the soft pot tray is to arrive in a completely embedded state, $V_{1}$ must satisfy the following:

$$
A_{3}=\frac{1}{2} M V_{1}^{2}=\int_{L-L_{0}}^{L} f_{2} x d x=\frac{1}{2} \frac{M}{L} \mu_{2} g\left(2 L-L_{0}\right) L_{0}
$$

where, $A_{3}=$ negative work arising from the friction between soft pot tray and embedded hard tray at $L_{0}(J)$.

Along with Equations (3)-(7), the conveyor belt $V_{0}$ for completely embedding the soft pot tray into the embedded hard tray must satisfy the following formula according to belt acceleration principles:

$$
\frac{1}{2} M V_{0}^{2}=-A_{1}+A_{2}+A_{3}
$$

Substitute Equation (3) into Equation (8) to obtain the following equation:

$$
V_{0}=\sqrt{\frac{g}{L}\left[-\mu_{1}\left(\frac{\mu_{2}}{\mu_{1}+\mu_{2}}\left(L-L_{0}\right)\right)^{2}+\mu_{2}\left(2 L-2 L_{0}-\frac{\mu_{2}}{\mu_{1}+\mu_{2}}\left(L-L_{0}\right)\right) \frac{\mu_{2}}{\mu_{1}+\mu_{2}}\left(L-L_{0}\right)+\mu_{2}\left(2 L-L_{0}\right) L_{0}\right]}
$$

According to the experimental measurements, $\mu_{1}$ is 0.8 (non-slip PVC high friction conveyor belt for conveyor belt) and $\mu_{2}$ is 0.6. The other parameters are as follows: soft pot tray length $L=600 \mathrm{~mm}$ and $L_{0}=130 \mathrm{~mm}$, and from Equation (9), get $V_{0}=$ $0.86 \mathrm{~m} / \mathrm{s}$.

To ensure that soft pot trays with sown seeds are completely embedded in hard trays, the minimum linear velocity $V_{0}$ of the conveyor belt is $0.86 \mathrm{~m} / \mathrm{s}$. This finding provided a theoretical base to optimize the conveyor-belt embedding mechanism design.

\subsection{Embedded-hard-tray automatic lowering mechanism and} transport mechanism

2.4.1 Composition of the mechanism

The embedded-hard-tray automatic lowering mechanism is a cube-type automatic tray-supplying mechanism, as shown in Figure 5. The length, width, and height of this mechanism are 600, 500, $400 \mathrm{~mm}$ respectively. It comprises electrically controlled steering gears (including steering-gear coupling, steering-gear fixing plates, a lifting head, a lifting shaft, and a frame). Two electrically controlled steering gears are separately mounted on steering-gear fixing plates on two sides of the frame, with two synchronized electrical circuits used to control the steering gears. A steering-gear coupling is used to link the steering gear and the lifting shaft, and the lifting head is mounted on the lifting shaft. In this study, to achieve a reasonably compact structure, the lifting-head angle and length were designed to be $90^{\circ}$ and $34 \mathrm{~mm}$, respectively. Furthermore, the distance between the two-axle centers was designed to be $370 \mathrm{~mm}$.

The transport mechanism is located at the bottom of the embedded-hard-tray automatic lowering mechanism. It comprises a transport axle, rubber roller, and axle sleeve. The movement of the transport structure is driven by $M_{2}$. To increase friction, the outer cylindrical surfaces of the rubber rollers have shallow grooves that are added during processing.

2.4.2 Work process of embedded-hard-tray automatic lowering mechanism

In accordance with the equipment working principles, the operation switch in the electrical control box is activated to restore the steering gear to its original position, with the lifting-head starting point at zero (see Figure 6a). When the soft pot tray touches the detection switch $A$, the electrical steering gear is activated. This turns the lifting shaft and drives the lifting head to raise all trays from the lowest-level embedded hard tray upward (see Figure 6b). Then, detection switch $C$ detects embedded hard trays as well as soft pot trays, the electrical steering gear operates again to drive the lifting head to lower the additional accumulated embedded hard trays downward (see Figure 6c). Once the next soft pot tray arrives, the above process is repeated to perform continuous operation.
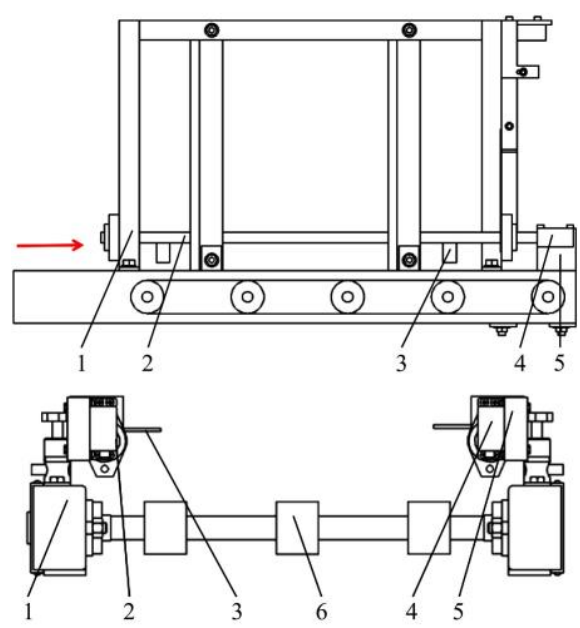

1. Frame 2. Lifting shaft 3. Lifting head 4. Electrical steering gear 5. Steering-gear fixing plates 6 . Transport mechanism

Figure 5 Embedded-hard-tray automatic lowering mechanism and transport mechanism
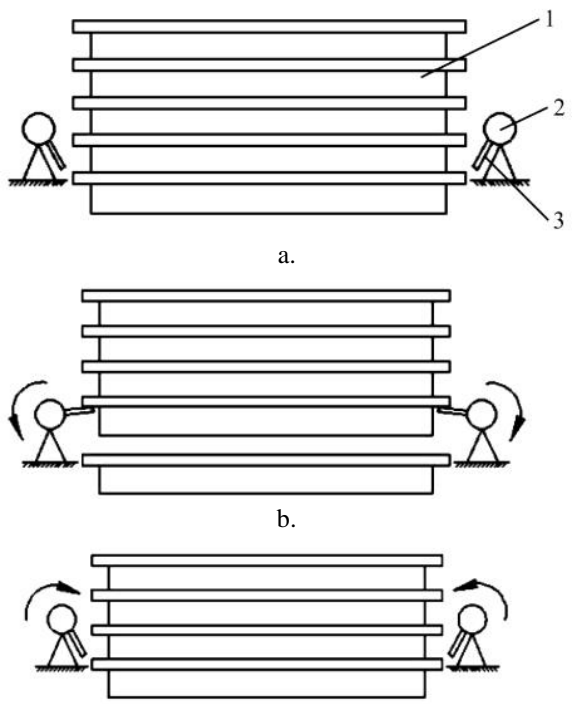

1.Stack of embedded hard trays 2 . Lifting shaft 3 . Lifting head

Figure 6 Work process analysis of embedded-hard-tray automatic lowering mechanism 


\subsection{Control system design}

2.5.1 Hardware design of control system

To achieve a stable and effective control system, a simplified control system was developed using the Arduino UNO R3 micro-controller as the primary controller. The system input/output (I/O) includes three switch inputs and three switch outputs. For micro-controller configuration, three switch signals of detectors $A, B$, and $C$ are output through one circuit (circuit 1) that synchronously controls the two steering gears. Furthermore, the output signals of circuit- 1 control relay $M_{1}$ and $M_{2}$. For the detection switch, an E18-D80NK model diffuse-reflection-type infrared photostatic switch is used. The optocoupler isolation relay uses a $5 \mathrm{~V}$ relay that supports high-and low-level triggering. Figure 7 shows the control system wiring diagram.

This study uses a $12 \mathrm{~V}$ direct current lithium battery as a power supply. We used the LM2596S step-down switching voltage regulator to first reduce the voltage from $12 \mathrm{~V}$ to $7 \mathrm{~V}$ before using the LM1117-5 low dropout voltage regulator chip to obtain a voltage of 3.3-5 V voltage to satisfy the voltage requirements for every module, the diagram of the power supply circuit is shown in Figure 8a. To improve the reliability of the control system, adding effective anti-interference measures in the process of designing the control system is necessary. The diagram of the signal acquisition circuit is shown in Figure 8b.

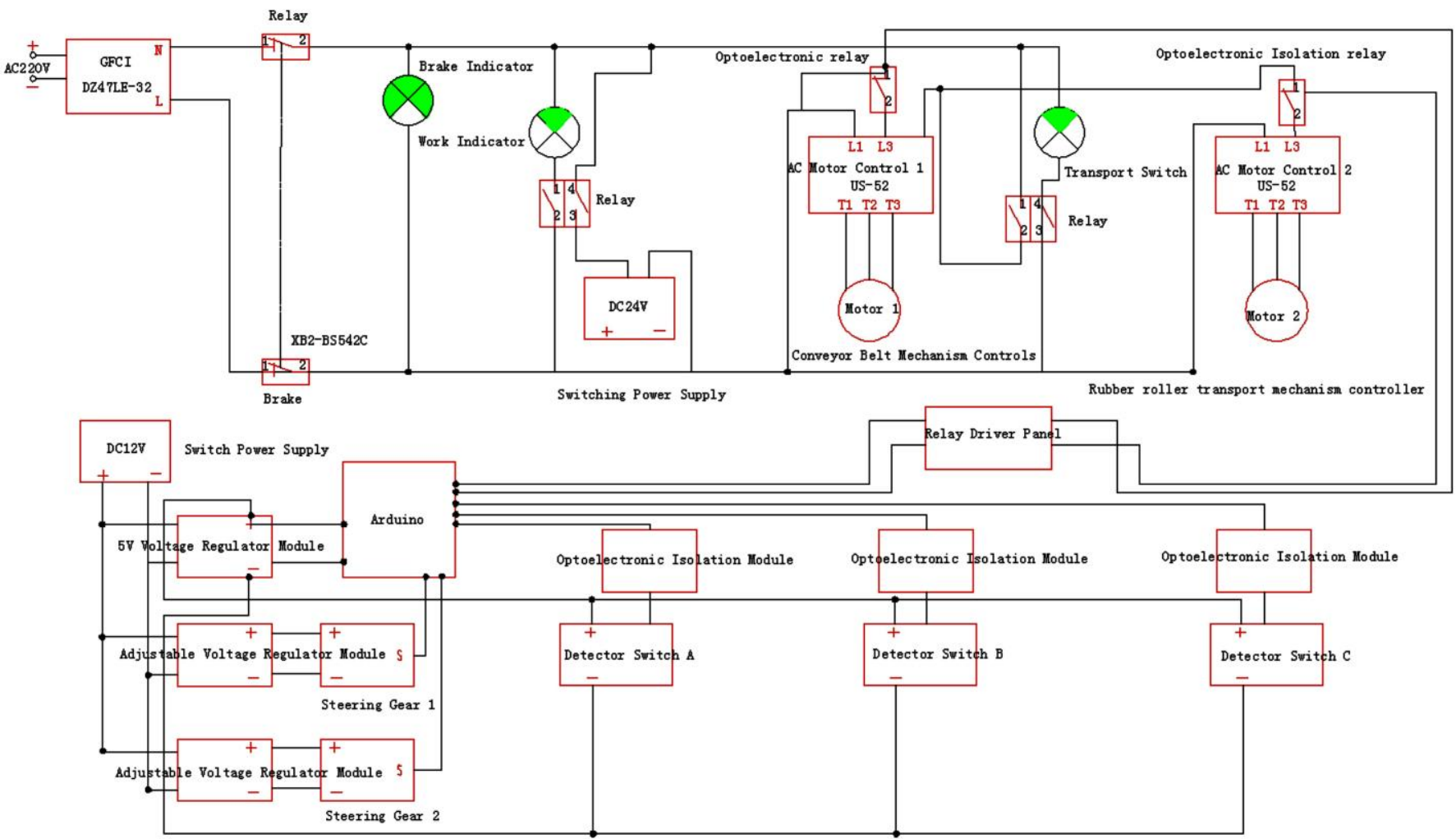

Figure 7 Control system wiring diagram
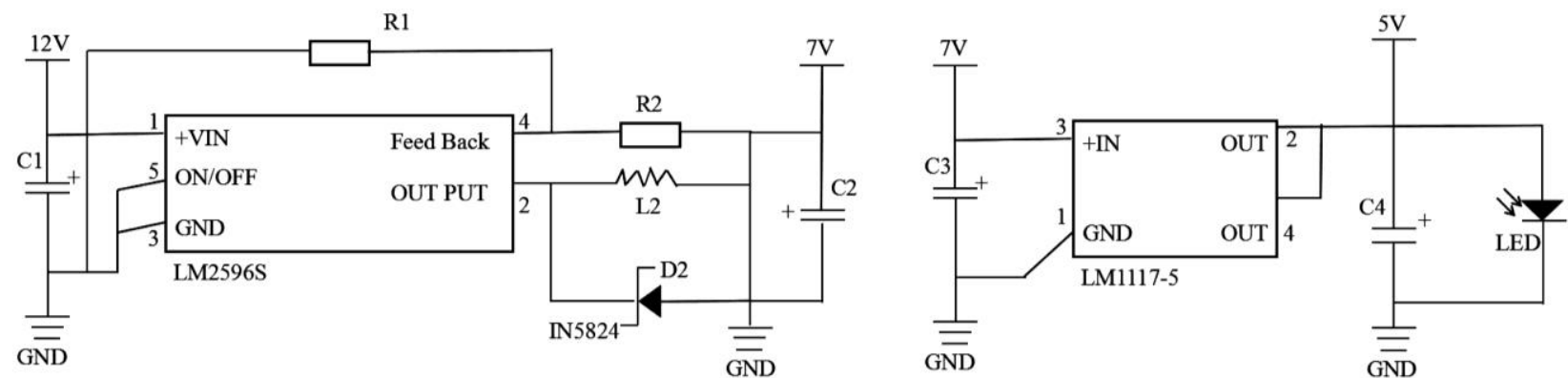

a. Diagram of the stabilized voltage supply circuit

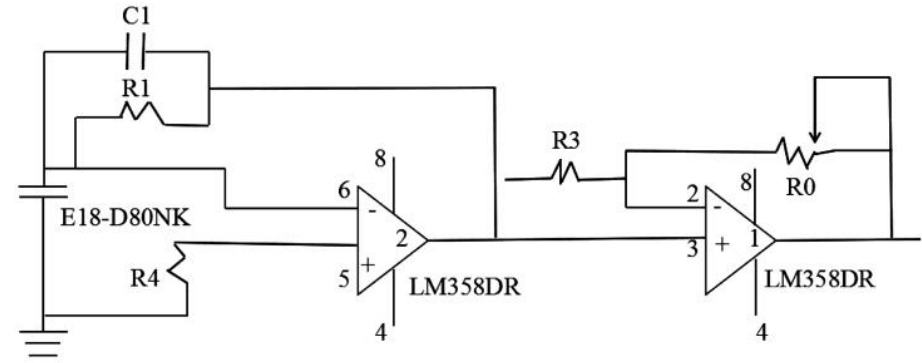

b. Diagram of the signal acquisition circuit

Figure 8 Hardware circuit design 
2.5.2 Process analysis of the delivery and embedding operation for soft pot trays

The process analysis of the delivery and embedding operation for soft pot trays is shown in Figure 9. The velocity of conveying the soft pot trays in the seedling nursery machine is set as $V^{\prime}(\mathrm{m} / \mathrm{s})$, the velocity of the conveying trays for the transport mechanism is $V_{2}$ $(\mathrm{m} / \mathrm{s})$, the length of the conveyor belt is $L_{c}(\mathrm{~m})$. The length of the conveying trays for the transport mechanism, $L g(\mathrm{~m})$, is $0.6 \mathrm{~m}$.

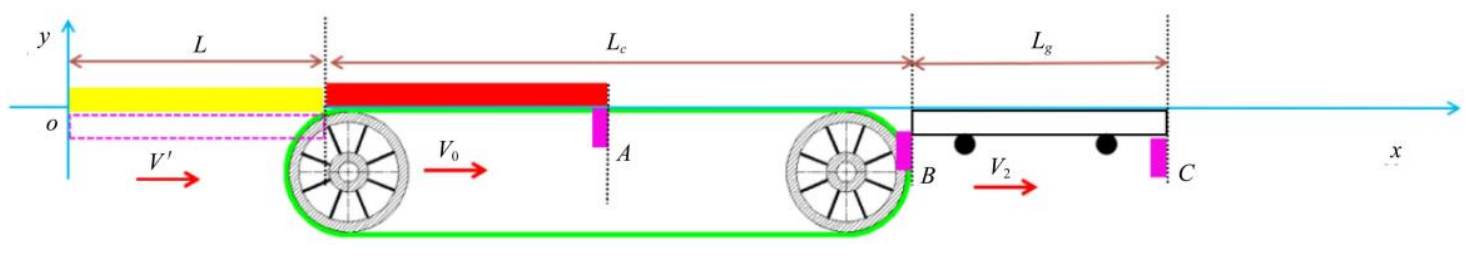

a.

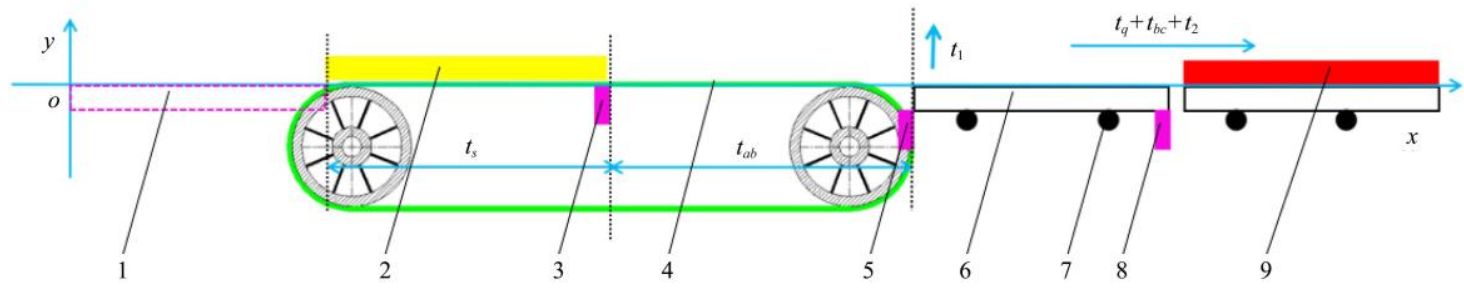

b.

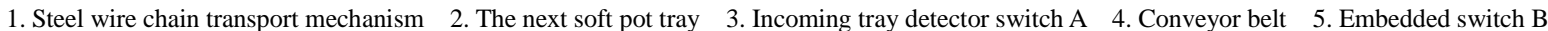
6. Hard tray 7. Transport mechanism $\quad 8$. Transport completion switch C 9. First soft pot tray and hard tray

Figure 9 Schematic of soft-pot-tray movement process

To ensure reliable operation of the embedded-hard-tray automatic lowering mechanism, the time $t_{a b}$ (s) taken by the first soft pot tray to reach embedded switch B from the incoming tray detector switch A must be greater than the time $t_{1}(\mathrm{~s})$ taken by lifting the accumulating hard trays and staying a hard tray using electronically controlled steering gear, i.e.,

$$
t_{a b} \geq t_{1}
$$

Then, the time $t_{s}(\mathrm{~s})$, at which the next soft pot tray arrives at the proximity switch A of the conveyor belt from the leaving point of the steel-wire-driven transport mechanism must be greater than the time $t_{q}(\mathrm{~s})$ of the previous soft pot tray embedded into the hard tray from the embedded switch $\mathrm{B}$, the time $t_{b c}(\mathrm{~s})$ taken by the transport mechanism to complete the delivery trays, and the time $t_{2}$ (s) taken by the electronically controlled steering gear completes the lowering and lifting of the hard tray and staying one hard tray, i.e.,

$$
t_{s}=\frac{L}{V^{\prime}} \geq t_{q}+t_{b c}+t_{2}
$$

For the seedling nursery machine driven by motor $M_{0}$ (see Figure 11), the transport speed $V^{\prime}$ is related to the productivity rate $Q$ (trays/h) of the seedling

$$
V^{\prime}=\frac{L Q}{3600}
$$

By simplifying the analysis, the average speed $\overline{V_{0}}(\mathrm{~m} / \mathrm{s})$ is approximately the same as $2 V_{0} / 3$, and therefore, the time $t_{q}$ must satisfy

$$
t_{q}=\frac{L}{\overline{V_{0}}}
$$

From Equations (11) to (13),

$$
V_{2}=\frac{L_{g}}{t_{b c}} \geq \frac{L_{g}}{t_{s}-t_{q}-t_{2}}=\frac{2 L_{g} Q V_{0}}{7200 V_{0}-3 Q L-2 Q V_{0} t_{2}}
$$

The length of the conveyor belt, $L_{c}$, must satisfy

$$
L_{c} \geq L+V_{0} t_{2}
$$

The electrical steering gear needs $0.23 \mathrm{~s}$ to rotate $90^{\circ}$, i.e. $t_{1}=0.23 \mathrm{~s}$ and $t_{2}=0.46 \mathrm{~s}$. The productivity of the seedling nursery machine is calculated to be 500 trays/h, i.e., the speed of conveying a soft pot tray is $V^{\prime}=0.083 \mathrm{~m} / \mathrm{s}$; the linear velocity of the conveyor belt is $V_{0} \geq 0.86 \mathrm{~m} / \mathrm{s}$, as obtained from the previous analysis. To ensure stable operation, the length of the conveyor belt can be calculated as $L_{c} \geq 1.2 \mathrm{~m}$ by using Equation (15). Using Equation (14), the minimum speed of the conveyor tray can be calculated as $V_{2} \geq 0.11 \mathrm{~m} / \mathrm{s}$.

To ensure continuous embedding operation of the soft pot trays, the length of the conveyor belt and the working parameters of the transport mechanism were determined. The length $L_{c}$ of the conveyor belt should be set to $1.2 \mathrm{~m}$, the velocity $V_{2}$ of the conveying trays for the transport mechanism should be set to $0.11 \mathrm{~m} / \mathrm{s}$.

2.5.3 Work flow of the control system

Figure 10 shows the control work flow during system operation.

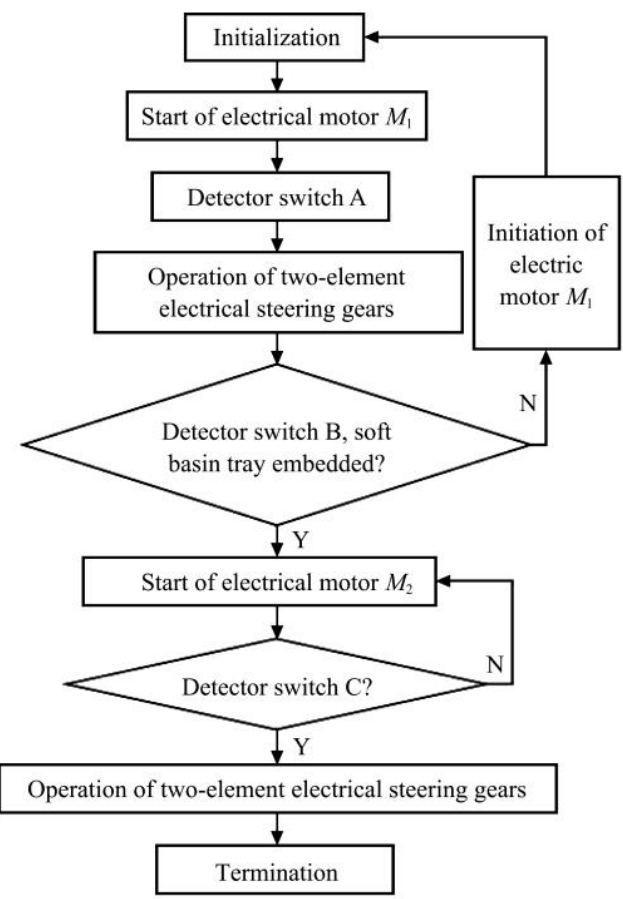

Figure 10 Control system work flowchart 


\section{Experiment and results}

To test the reliability and stability of the embedded-hard-tray automatic lowering mechanism and to validate the theoretical analysis, the tray placement success rate, tray embedding success rate, and seed exposure rates were used as performance evaluation indicators in an experimental study of the soft-pot-tray automatic embedding system ${ }^{[22,23]}$.

\subsection{Experimental indications}

The tray placement success rate is defined as the ratio of successful tray placements to the total number of tray placements. The tray placement success rate is calculated as follows:

$$
\mathrm{S}=\frac{K_{1}}{K_{0}} \times 100 \%
$$

where, $S$ is successful tray placement rate, $\% ; K_{1}$ is the number of successful tray placements; $K_{0}$ is the number of placed trays.

The tray embedding success rate is defined as the ratio of the number of successful tray embeddings to the total number of tray embeddings. The tray embedding success rate is calculated as follows:

$$
Y=\frac{Q_{1}}{Q_{0}} \times 100 \%
$$

where, $Y$ is the successful tray embedding rate, $\% ; Q_{1}$ is the number of successful tray embeddings; $Q_{0}$ is the total number of tray embeddings.

If the conveyor belt $V_{0}$ is too low, the soft pot tray will not be completely embedded into the embedded hard tray. If the conveyor belt $V_{0}$ is too high, collision-like contact or vibration will occur between the soft pot tray and embedded-hard-tray end portion, causing exposure of the sown seeds and influencing the seedling cultivation quality. Thus, the seed exposure rate was used to measure the system performance; it is defined as the ratio of the number of exposed seeds to the number of holes in the soft pot tray during system operation. The seed exposure rate is calculated as follows:

$$
W=\frac{P_{1}}{P_{0}} \times 100 \%
$$

where, $W$ is seed exposure rate, $\% ; P_{1}$ is number of seed exposure holes; $P_{0}$ is the number of holes in the soft pot tray.

\subsection{Experimental procedure}

The test was divided into three steps. Firstly, the tray placement success rate was used as an evaluation indicator by taking the different production rates as the experimental factor to test the performance of the embedded-hard-tray automatic lowering mechanism. Secondly, the influence of different conveyor belt $V_{0}$ on the soft-pot-tray embedding success and seed exposure rates was examined. Thirdly, the different production rates on the soft-pot-tray embedding success and seed exposure rates were examined.

The experiments were performed at the South China Agricultural University College of Engineering Laboratory; the site and equipment are shown in Figure 11. Before the experiment, a batch of embedded hard trays and soft pot trays was assembled. As the deformation of the embedded hard trays and soft pot trays influences system operational stability, good trays were selected for these operations. During the experiment, a Jiyu Huayu 2BB-14 rice seedling pot-tray nursery machine was used to sow the cultivated seedlings in the soft pot trays. After sowing was complete, each soft pot tray continued to the pot-tray automatic embedding system for testing. The soil used in this experiment was post-crush processed rice-paddy-field sandy loam that had been filtered by three sieves and that had $14 \%-20 \%$ water content. Peiza taifeng hybrid rice with sprout lengths of $\leq 2 \mathrm{~mm}$ and water content of up to $24 \%$ was used $^{[23-25]}$.

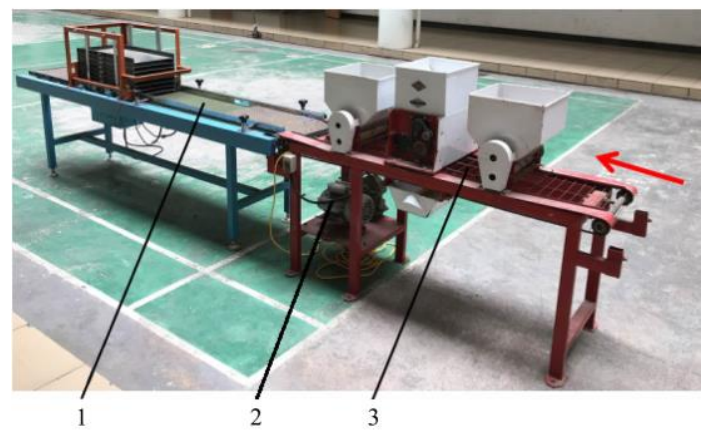

1. Soft-pot-tray automatic embedding system 2. Jiyu Huayu 2BB-14 rice seedling pot-tray nursery machine 3 . Motor $M_{0}$

Figure 11 Experiment equipment and site

\subsection{Experimental results and discussion}

In accordance with the working principles of the embedded-hard-tray automatic lowering mechanism, the production rate greatly influences its performance indicator(s). Therefore, the production rate was used as the variable when performing the experimental study. The position-limiting mechanisms of both sides and the conveyor-belt embedding mechanism were set to normal working conditions, and conveyor belt $V_{0}$ was set to $0.89 \mathrm{~m} / \mathrm{s}$. Currently, the rice tray nursing seedling planter production rate is generally $400-800$ trays $/ \mathrm{h}$. During the experiment, different production levels were selected. Testing with 100 embedded hard trays was performed, where each tray was placed into the embedded-hard-tray automatic lowering mechanism 10 times. The production rate was adjusted to four values: $400,500,600$, and 700 trays/h. The number of trays that could be normally raised and lowered was counted, and the experiment was repeated seven times for each production level. The experimental results are listed in Table 1 and the change trends are shown in Figure 12.

Table 1 and Figure 12 shows that the tray placement success rate decreased with increasing productivity. Therefore, to satisfy the technological requirements of the embedded-hard-tray lowering mechanism operation and to reduce vibration and impact effects, the productivity during the operation was set to 500 trays $/ \mathrm{h}$, this

\begin{tabular}{|c|c|c|c|c|c|c|c|c|c|}
\hline \multirow{2}{*}{$\begin{array}{l}\text { Production } \\
\text { rate } \\
\text { /trays } \cdot \mathrm{h}^{-1}\end{array}$} & \multicolumn{7}{|c|}{ Sequence } & \multirow{2}{*}{$\begin{array}{c}\text { Average tray } \\
\text { placement } \\
\text { success rate from } \\
\text { seven runs/\% }\end{array}$} & \multirow[b]{2}{*}{$\begin{array}{l}\text { Standard } \\
\text { deviation }\end{array}$} \\
\hline & $\begin{array}{c}\text { Run } 1 \\
\text { Tray placement } \\
\text { success rate/\% }\end{array}$ & $\begin{array}{c}\text { Run } 2 \\
\text { Tray placement } \\
\text { success rate/\% }\end{array}$ & $\begin{array}{c}\text { Run } 3 \\
\text { Tray placement } \\
\text { success rate/\% }\end{array}$ & $\begin{array}{c}\text { Run } 4 \\
\text { Tray placement } \\
\text { success rate/\% }\end{array}$ & $\begin{array}{c}\text { Run } 5 \\
\text { Tray placement } \\
\text { success rate } / \%\end{array}$ & $\begin{array}{c}\text { Run } 6 \\
\text { Tray placement } \\
\text { success rate/\% }\end{array}$ & $\begin{array}{c}\text { Run } 7 \\
\text { Tray placement } \\
\text { success rate/\% }\end{array}$ & & \\
\hline 400 & 100 & 100 & 100 & 100 & 100 & 100 & 100 & 100 & 0 \\
\hline 500 & 100 & 100 & 99 & 100 & 99 & 100 & 100 & 99.7 & 0.0048 \\
\hline 600 & 98 & 97 & 99 & 100 & 98 & 100 & 99 & 98.7 & 0.0111 \\
\hline
\end{tabular}
ensured $>99 \%$ successful tray placement rate.

Table 1 Successful tray placement rates under different production rates 


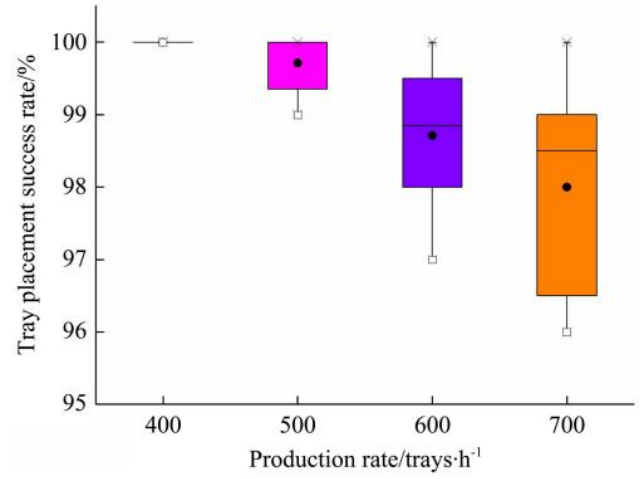

Figure 12 Change trends of successful tray placement under different production rates

Adjusting the embedded-hard-tray automatic lowering mechanism to the normal operational state, the production rate was set to 500 trays $/ \mathrm{h}$. The conveyor belt $V_{0}$ was separately adjusted to $0.8,0.83,0.86,0.89,0.92$, and $0.95 \mathrm{~m} / \mathrm{s}$ and experiments were conducted. Again, 100 seedling trays were tested for each linear velocity and each tray was placed on the embedded-hard-tray automatic lowering mechanism 10 times. Each $V_{0}$ value was tested seven times. The experiment results for the tray embedding success rate and seed exposure rate are shown in Table 2 and the corresponding change trends are shown in Figures 13.

Table 2 and Figure 13 shows that the conveyor belt $V_{0}$ obviously influences the tray embedding success rate. When the conveyor belt $V_{0}$ was $<0.86 \mathrm{~m} / \mathrm{s}$, the tray embedding success rate was $\leq 6 \%$. The experiment revealed that a relatively large part of the soft pot tray remains outside the embedded hard tray in this case (see Figure 14a); therefore, the technological requirements of pot-tray embedding operations were not satisfied. However, when the conveyor belt $V_{0}$ exceeded $0.86 \mathrm{~m} / \mathrm{s}$, the tray embedding success rate increased with the conveyor belt $V_{0}$; the experiment and theoretical analysis results for this case match. Finally, when the conveyor belt $V_{0}$ was set to $\geq 0.92 \mathrm{~m} / \mathrm{s}$, the tray embedding success rate was $100 \%$ (see Figure 14b). With increased conveyor belt $V_{0}$, the seed exposure rate gradually increases. When the conveyor belt $V_{0}$ is $\geq 0.95 \mathrm{~m} / \mathrm{s}$, the impact of the soft pot tray on the embedded-hard-tray end portion increases and the seed exposure rate reaches $\geq 1.6 \%$. However, when the conveyor belt $V_{0}$ is $0.92 \mathrm{~m} / \mathrm{s}$, the seed exposure rate is $\leq 0.98 \%$; this meets the technological requirements of the system for actual operation.

Table 2 Experiment results for tray embedding success rate and seed exposure rate under different conveyor-belt linear velocities

\begin{tabular}{|c|c|c|c|c|c|c|c|c|c|}
\hline \multirow[b]{2}{*}{$\begin{array}{c}\text { Conveyor-belt } \\
\text { speed } \\
/ \mathrm{m} \cdot \mathrm{s}^{-1}\end{array}$} & \multicolumn{7}{|c|}{ Sequence } & \multirow{2}{*}{$\begin{array}{l}\text { Average tray } \\
\text { embedding success } \\
\text { rate from seven } \\
\text { runs/Average seed } \\
\text { exposure rate from } \\
\text { seven runs/\% }\end{array}$} & \multirow[b]{2}{*}{$\begin{array}{l}\text { Standard } \\
\text { deviation }\end{array}$} \\
\hline & $\begin{array}{c}\text { Run } 1 \\
\text { Tray embedding } \\
\text { success rate/ } \\
\text { Seed exposure } \\
\text { rate } / \%\end{array}$ & $\begin{array}{c}\text { Run } 2 \\
\text { Tray embedding } \\
\text { success rate/ } \\
\text { Seed exposure } \\
\text { rate/\% }\end{array}$ & $\begin{array}{c}\text { Run } 3 \\
\text { Tray embedding } \\
\text { success rate/ } \\
\text { Seed exposure } \\
\text { rate/\% }\end{array}$ & $\begin{array}{c}\text { Run } 4 \\
\text { Tray embedding } \\
\text { success rate/ } \\
\text { Seed exposure } \\
\text { rate/\% }\end{array}$ & $\begin{array}{c}\text { Run } 5 \\
\text { Tray embedding } \\
\text { success rate/ } \\
\text { Seed exposure } \\
\text { rate/\% }\end{array}$ & $\begin{array}{c}\text { Run } 6 \\
\text { Tray embedding } \\
\text { success rate/ } \\
\text { Seed exposure } \\
\text { rate/\% }\end{array}$ & $\begin{array}{c}\text { Run } 7 \\
\text { Tray embedding } \\
\text { success rate/ } \\
\text { Seed exposure } \\
\text { rate/\% }\end{array}$ & & \\
\hline 0.8 & $3 / 0$ & $4 / 0$ & $4 / 0$ & $2 / 0$ & $3 / 0.24$ & $2 / 0$ & $4 / 0.24$ & $3.1 / 0.068$ & $0.0090 / 0.0011$ \\
\hline 0.86 & $98 / 0.25$ & $99 / 0$ & $98 / 0.50$ & $97 / 0.49$ & $97 / 0$ & $99 / 0.25$ & $97 / 0.49$ & $97.8 / 0.28$ & $0.0089 / 0.0022$ \\
\hline 0.89 & $99 / 0.73$ & $99 / 0.25$ & $99 / 0.73$ & $100 / 0.49$ & $97 / 0.24$ & $97 / 0.25$ & $99 / 0.45$ & $98.5 / 0.45$ & $0.0113 / 0.0021$ \\
\hline 0.92 & $100 / 1.23$ & $100 / 0.73$ & $100 / 1.23$ & $100 / 1.23$ & $100 / 0.73$ & $100 / 0.49$ & $100 / 0.98$ & $100 / 0.98$ & $0 / 0.0032$ \\
\hline 0.95 & $100 / 1.47$ & $100 / 1.97$ & $100 / 1.23$ & $100 / 1.72$ & $100 / 1.47$ & $100 / 1.72$ & $100 / 1.6$ & $100 / 1.6$ & $0 / 0.0024$ \\
\hline
\end{tabular}
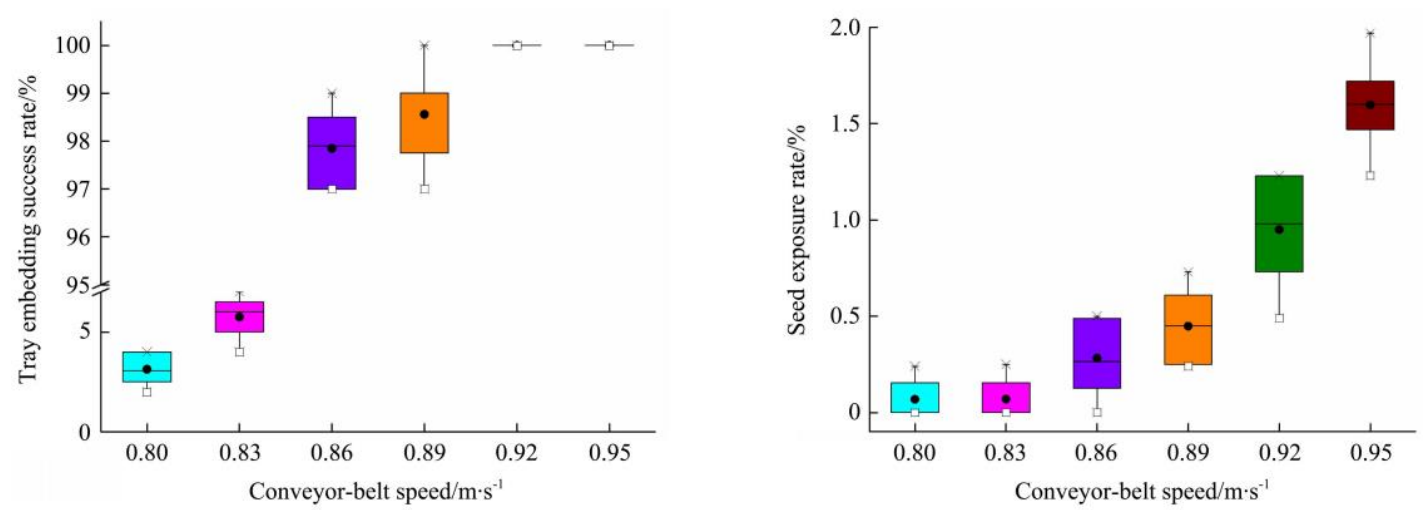

Figure 13 Change trends in tray embedding success rate and seed exposure rate
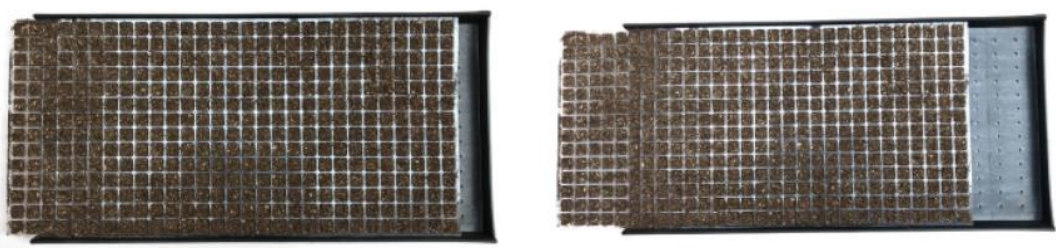

a. Failed tray embedding and seed exposure

Figure 14 Photos of experimental results

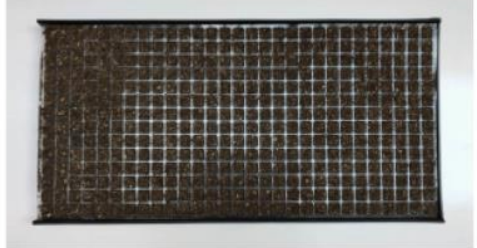

b. Successful tray embedding
To further examine the performance of the entire machine, and to verify the reliability and stability of key components under different production rates. The conveyor belt $V_{0}$ was set to $0.92 \mathrm{~m} / \mathrm{s}$, the productivity was separately adjusted to 600 and 
700 trays/h and experiments were conducted. Again, 100 seedling trays were tested for each productivity and each tray was placed on the embedded-hard-tray automatic lowering mechanism ten times. Each productivity was tested seven times. The experiment results for the tray embedding success rate and seed exposure rate are shown in Table 3 and the corresponding change trends are shown in Figures 15.

Table 3 Experiment results for tray embedding success rate and seed exposure rate under different production rates

\begin{tabular}{|c|c|c|c|c|c|c|c|c|c|}
\hline \multirow[b]{2}{*}{$\begin{array}{l}\text { Production } \\
\text { rate } \\
\text { / trays } \cdot \mathrm{h}^{-1}\end{array}$} & \multicolumn{7}{|c|}{ Sequence } & \multirow[b]{2}{*}{$\begin{array}{c}\text { Average tray } \\
\text { embedding success } \\
\text { rate from seven } \\
\text { runs/Average seed } \\
\text { exposure rate from } \\
\text { seven runs/\% }\end{array}$} & \multirow[b]{2}{*}{$\begin{array}{l}\text { Standard } \\
\text { deviation }\end{array}$} \\
\hline & $\begin{array}{c}\text { Run } 1 \\
\text { Tray embedding } \\
\text { success rate } \\
\text { /Seed exposure } \\
\text { rate/\% }\end{array}$ & $\begin{array}{c}\text { Run } 2 \\
\text { Tray embedding } \\
\text { success rate } \\
\text { /Seed exposure } \\
\text { rate/\% }\end{array}$ & $\begin{array}{c}\text { Run } 3 \\
\text { Tray embedding } \\
\text { success rate } \\
\text { /Seed exposure } \\
\text { rate/\% }\end{array}$ & $\begin{array}{c}\text { Run } 4 \\
\text { Tray embedding } \\
\text { success rate } \\
\text { /Seed exposure } \\
\text { rate/\% }\end{array}$ & $\begin{array}{c}\text { Run } 5 \\
\text { Tray embedding } \\
\text { success rate } \\
\text { /Seed exposure } \\
\text { rate/\% }\end{array}$ & $\begin{array}{c}\text { Run } 6 \\
\text { Tray embedding } \\
\text { success rate } \\
\text { /Seed exposure } \\
\text { rate/\% }\end{array}$ & $\begin{array}{c}\text { Run } 7 \\
\text { Tray embedding } \\
\text { success rate } \\
\text { /Seed exposure } \\
\text { rate/\% }\end{array}$ & & \\
\hline 600 & $98 / 1.47$ & $96 / 1.23$ & $98 / 0.73$ & $100 / 1.97$ & $99 / 1.23$ & $100 / 1.47$ & $99 / 1.23$ & $98.5 / 1.33$ & $0.0139 / 0.0037$ \\
\hline
\end{tabular}
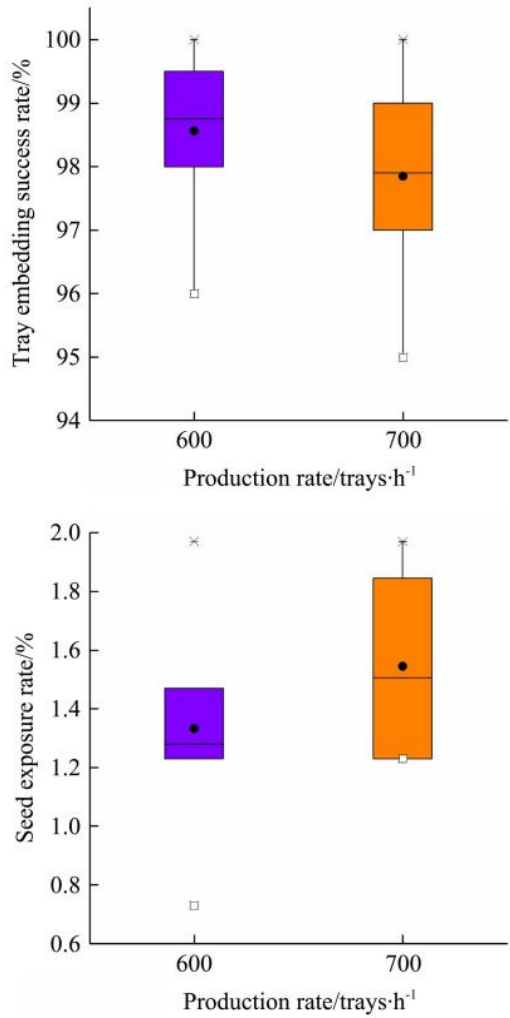

Figure 15 Change trends in tray embedding success rate and seed exposure rate

Table 3 and Figure 15 shows that the seed exposure rate was increased and the tray embedding success rate was reduced slightly as the productivity increases when the linear velocity $V_{0}$ of the conveyor belt was $0.92 \mathrm{~m} / \mathrm{s}$. When the productivity was set to 700 trays/h, the tray embedding success rate was $\geq 97.8 \%$ and the seed exposure rate was $\geq 1.54 \%$. The analysis of variance shows that the fluctuation of experimental data is very small. The reliability and stability of the whole machine can be ensured.

\section{Conclusions}

(1) A soft-pot-tray automatic embedding system was designed for a light-economical pot seedling nursery machine in this study. The control system was developed in the Arduino programming environment. The structures of the conveyor-belt embedding mechanism, embedded-hard-tray automatic lowering mechanism, and transport mechanism were proposed to achieve desired functions.

(2) The system dynamics was analyzed and an equation was developed to describe the embedding of the soft pot trays into the embedded hard trays. The minimum conveyor-belt linear velocity required for a complete soft-pot-tray embedding into the embedded hard tray was found to be $0.86 \mathrm{~m} / \mathrm{s}$. The required length of the conveyor belt was $1.2 \mathrm{~m}$ for continuous embedding operations. The velocity of conveying trays for the transport mechanism should be set to $0.11 \mathrm{~m} / \mathrm{s}$.

(3) A prototype was built and the proposed system was tested in the laboratory condition. The experimental results showed that the tray embedding success rate was $100 \%$ and the seed exposure rate was less than $1 \%$ with a production rate of 500 trays $/ \mathrm{h}$ and a conveyor-belt linear velocity of $0.92 \mathrm{~m} / \mathrm{s}$. The experimental findings supported the dynamics analysis and warranted the performance of the soft-pot-tray automatic embedding system.

\section{Acknowledgements}

The authors gratefully acknowledge the financial support from the National Key Research and Development Program of China (Grant No.2018YFD0700703); National Natural Science Foundation of China (Grant No.51675188) and the Earmarked Fund for Modern Agro-industry Technology Research System (Grant No.CARS-01-43).

\section{[References]}

[1] Bai R P. Thinking of accelerating solutions to bottleneck restrictions of rice planting mechanization. Journal of CSAM, 2016; 37(12): 1-5, 47. (in Chinese)

[2] Ma G H, Yuan L P. Hybrid rice achievements, development and prospect in China. Journal of Integrative Agriculture, 2015; 14(2): 197-205.

[3] Li Z H, Ma X, Liang L, Qi L. Analysis of influence factors on development of rice planting mechanization based on grey correlation analysis. Journal of Basic Science and Engineering, 2013; 21(6): 1167-1177. (in Chinese)

[4] Li L H, Wang C, Zhang X Y, Sarker K K. Mechanized cultivation technology of seedling-growing bowl tray made of paddy-straw and its effects on rice production. Int J Agric \& Biol Eng, 2015; 24(3): 97-103.

[5] Hu ZX, Tian Y, Xu Q S. Review of extension and analysis on current status of hybrid rice in China. Hybrid Rice, 2016; 31(2): 1-8.

[6] Singh R S, Gite L P. Technological change in paddy production: A comparative analysis of traditional and direct seeding methods of cultivation. AMA-Agricultural mechanization in Asia Africa and Latin America, 2012; 43(3): 41-46.

[7] Konishi T, Horio M, Yoshida S. Development of high performance rice transplanter. Journal of the Japanese Society of Agricultural Machinery, 2010; 51(5): 89-95.

[8] Yu X X, Zhao Y, Chen B C, Zhou M L, Zhang H, Zhang Z C. Current situation and prospect of transplanter. Transactions of the CSAM, 2014; 45(8): 44-53. (in Chinese)

[9] Yi S J, Liu Y F, Wang C, Tao G X. Experimental study on the performance of bowl-tray rice precision seeder. Int J Agric \& Biol Eng, 2014; 7(1): $17-25$.

[10] Gaikwad B B, Sirohi N P S. Design of a low-cost pneumatic seeder for nursery plug trays. Biosystems Engineering, 2008; 99: 322-329.

[11] Du L H, Yu G H, Zhang G F, Li G, Liu D Q. Design and experiment of 
vertically feeding-seedling device for pot-blanket seedling based on high-speed rice transplanter. Transactions of the CSAE, 2014; 30(14): 17-25. (in Chinese)

[12] Movahedi E, Rrzvani M, Hemmat A. Design, development and evaluation of a pneumatic seeder for automatic planting of seeds in cellular trays. Journal of Agricultural Machinery, 2016; 4(1): 65-72.

[13] Ma X, Xie J F, Qi L, Liang Z W, Kuang J X, Tan Y X. Subsoil compaction device for rice seedling nursery planter of bowl seedling tray. Transactions of the CSAM, 2014; 45(8): 54-60. (in Chinese)

[14] Zhou H B, Ma X, Yao Y L. Research advances and prospects in the seeding technology and equipment for tray nursing seedlings of rice. Transactions of the CSAE, 2008; 24(4): 301-306. (in Chinese)

[15] Jin, X, Li D Y, Ma H, Ji J T, Zhao K X, Pang, J. Development of single row automatic transplanting device for potted vegetable seedlings. Int $\mathrm{J}$ Agric \& Biol Eng, 2018; 11(3): 67-75.

[16] Forleo M B, Palmieri N, Suardi A, Coaloa D. The eco-efficiency of rapeseed and sunflower cultivation in Italy. Joining environmental and economic assessment. Journal of Cleaner Production, 2018; 172: 3138-3153.

[17] Kumar G V P, Raheman H. Automatic feeding mechanism of a vegetable transplanter. Int J Agric \& Biol Eng, 2012; 5(2): 20-27.

[18] Yu H F Y, Tsung C L, Ting Y L, Chang C L, Wei C C, Ta T L. An automated growth measurement system for leafy vegetables. Biosystems Engineering, 2014; 117: 43-50.

[19] Hector E, Glenn J F. Automated registration of hyperspectral images for precision agriculture. Computers and electronics in agriculture, 2005; 47 : 103-119.

[20] Iseki Co., Ltd. Automatic supply device for trays for sowing machine.
Japanese patent: 60-199304, 1985-10-08.

[21] Kubota Agriculture Machinery Co., Ltd. Trays automatic supply apparatus Japanese patent: 62-87008, 1987-04-21.

[22] Tan Y X. Study on pneumatic type automatic tray feeder and automatic tray stacker. Guangzhou: South China Agricultural University, 2015. (in Chinese )

[23] Ma X, Chen L T, Huang G, Qi L, Lin S M, Lu Q. Electric controlled automatic tray feeding device for hard and soft tray of rice precision seeding for nursing seedlings planter. Transaction of the CSAM, 2017; 48(6): 41-49. (in Chinese )

[24] Zhao Z, Wu Y F, Yin J J, Tang Z. Monitoring method of rice seeds mass in vibrating tray for vacuum-panel precision seeder. Computers and Electronics in Agriculture, 2015; 114: 25-31.

[25] Tan S Y, Ma X, Wu L L, Li Z H, Liang Z W. Estimation on hole seeding quantity of super hybrid rice based on machine vision and BP neural network. Transactions of the CSAE, 2014; 30: 201-208. (in Chinese)

[26] Rogalski A. Recent progress in infrared detector technologies. Infrared Physics \& Technology, 2011; 54(3): 136-154.

[27] Caprara C, Pezzi F. Evaluation of quality of harvest and mechanical aspects related to beater adjustments in mechanical grape harvesting.Trans of the ASABE, 2014; 57(4): 991-997.

[28] Willis J R, Milton G W. On modifications of Newton's second law and linear continuum elastodynamics. Proceedings of the Royal Society A: Mathematical, Physical and Engineering Sciences, 2007; 463(2079): 855-880.

[29] Ratnayake R M C, Balasoriya B M C P. Re-Design, fabrication, and performance evaluation of manual conical cylinder seeder: A case study.Applied Engineering in Agriculture, 2013; 29(2): 139-147. 